LIVER

\title{
Improved characterisation of histologically proven liver tumours by contrast enhanced ultrasonography during the portal venous and specific late phase of SHU 508A
}

\author{
C F Dietrich, A Ignee, J Trojan, C Fellbaum, G Schuessler
}

Gut 2004;53:401-405. doi: 10.1136/gut.2003.026260

See end of article for authors' affiliations

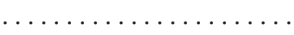

Correspondence to:

Dr C F Dietrich, Second

Department of Internal Medicine, Caritas Hospital Bad Mergentheim, Uhlandstr 7, D-97980 Bad Mergentheim, Germany; christoph.dietrich@ ckbm.de

Accepted for publication 22 September 2003

\begin{abstract}
Purpose: Ultrasound is reported to be relatively unreliable in the characterisation of liver tumours. The purpose of this study was to assess the ability of contrast enhanced phase inversion ultrasound (PIUS), a new highly sensitive contrast specific technique, performed during the liver specific phase of Levovist, to differentiate between benign and malignant lesions of the liver.

Patients and methods: A total of 174 patients with histologically proven liver tumours were prospectively examined with conventional B mode ultrasound and two minutes after intravenous bolus injection of SHU 508A (Levovist). The examination technique comprised: Siemens Sonoline Elegra, phase inversion harmonic imaging (ECI); high mechanical index (1.2-1.7) using a delayed two minute post contrast scanning technique.

Results: In all patients with malignant disease, hypoechoic contrast enhancement was seen during the portal venous phase, and convincing but variably less demarcated in 13 patients with hepatocellular carcinoma compared with all patients with liver metastases. The liver tumours proved to be histologically benign in 95 patients and malignant in 79 patients. Homogenous contrast enhancement with a mainly isoechogenic appearance in the portal venous and liver specific late phase was seen in almost all patients with benign liver lesions with the exception of one patient with an inflammatory pseudotumour of the liver and five patients with abscesses. These six exceptions all demonstrated a hypoechoic appearance in the portal venous and liver specific late phase.

Discussion: The ability of unenhanced ultrasonography to characterise liver disease is known to be limited. PIUS performed during the portal venous and liver specific late phase of Levovist may differentiate between benign and malignant liver tumours in most cases, with the exception of, for example, abscesses, scars, necrosis, cysts, and calcifications, which need to be excluded clinically and by conventional B mode ultrasonography.
\end{abstract}

Abbreviations: US, ultrasonography; $\mathrm{CT}$, computed tomography; $\mathrm{MRI}$, magnetic resonance imaging; PIUS, phase inversion ultrasound; HCC,

hepatocellular carcinoma; FNH, focal nodular hyperplasia

the first time that PIUS has been evaluated in a large number of patients with histologically proven benign and malignant liver tumours.

The purpose of this prospective study was to assess if PIUS, a new highly sensitive contrast specific technique performed during the portal venous and liver specific late phase of Levovist, can differentiate between benign and malignant liver tumours and improve the characterisation of liver tumours in comparison with unenhanced sonography.

\section{PATIENTS AND METHODS}

Patients

During a period of approximately two years, 174 patients (94 females and 80 males; median age 54 years (range 7-80)) were included in the current prospective study. The aetiology of the lesions was confirmed by liver biopsy in all patients.

Liver biopsy was performed close to the US examination in all patients with malignant lesions and in most patients with benign lesions $(<2$ weeks in all patients enrolled into the study). In addition, in all patients with benign liver tumours, the size of the lesion was stable during the interval between liver biopsy and the study. and MRI. It is of special interest that PIUS may also detect subcentimetre metastases and small hepatocellular carcinomas (HCCs)..$^{3-6}$

In contrast with recent radiological studies that involved few patients with benign lesions, mainly using contrast enhanced CT techniques and MRI as the gold standard, this is 
The analysis was made on a "lesion by lesion" basis. In patients with multiple lesions, we examined the lesion which had been diagnosed by liver biopsy and histology.

Reference imaging examinations (for example, CT, MRI, and scintigraphy) were performed as part of the clinical workup of the patients-in several cases outside our institution-and not for the purpose of this study. The standard practice used in our department included two different image procedures (US/CT/MRI) with inconclusive findings before liver biopsy.

Institutional Board approval and oral informed consent according to the ethical guidelines of Helsinki was obtained from all patients after informing them of the purpose and aim of the study before the US examination was started. Patient characteristics are summarised in table 1 .

\section{Methods}

\section{Fundamental US examination technique}

In all patients, conventional B mode US was performed, indicating non-conclusive results. Baseline US scans were performed using a Sonoline Elegra platform (Siemens Ultrasound Division, Issaquah, Washington, USA) with a 3.5 MHz and 7 MHz multifrequency transducer. All pre- and post-contrast examinations were carried out by the same examiner (CFD) but image analysis was performed by two examiners. Each patient underwent a conventional sonogram of the liver in fundamental B mode in longitudinal and transverse sections, with individually optimised scanner settings, as recently described..$^{7-9}$ In addition, power Doppler US of focal lesions using adequate low pulse repetition frequency was performed in all patients. Tumour vascularity was defined as hypervascular, isovascular, or hypovascular in comparison with the surrounding liver parenchyma. The depth of the lesion was $<12 \mathrm{~cm}$ in all patients.

\section{Contrast enhancing agent SHU 508A (Levovist)}

SHU 508A (Schering AG, Berlin, Germany) is a US contrast agent consisting of galactose microparticles (99.9\%) and palmitic acid $(0.1 \%)$ with a microbubble diameter of less than $7 \mu \mathrm{m}$ in approximately $97 \%$ of particles. After intravenous injection, Levovist circulates for several minutes in the general blood pool, with a liver specific late phase starting within two minutes of injection.

Table 1 Demographic profile and tumour size in patients with histologically proven liver tumours

\begin{tabular}{ll}
\hline Characteristic & \\
\hline Demography & \\
No (M/F) & $80 / 94$ \\
Mean age (yr) & $52(15)[7-80]$ \\
Benign liver lesions $(n=95)($ mean size $(\mathrm{mm}))$ & $41(24)[8-130]$ \\
Focal nodular hyperplasia $(n=36)$ & $51(18)[20-110]$ \\
Haemangioma $(n=31)$ & $37(31)[8-130]$ \\
Adenoma $(n=10)$ & $40(20)[15-80]$ \\
Abscess $(n=5)$ & $40(24)[10-80]$ \\
Microhamartomas $(n=4)$ & $12(2)[9-15]$ \\
Focal steatosis $(n=4)$ & $32(5)[25-38]$ \\
Nodular regenerative hyperplasia $(n=1)$ & 40 \\
Hyperregenerative nodule $(n=1)$ & 35 \\
Focal biliary cirrhosis $(n=1)$ & 25 \\
Bacillary angiomatosis $(n=1)$ & 50 \\
Inflammatory pseudotumour $(n=1)$ & 40 \\
Malignant liver lesions $(n=79)($ mean size $(\mathrm{mm}))$ & $40(21)[7-120]$ \\
Metastatic liver tumour $(n=37)$ & $33(12)[10-75]$ \\
Hepatocellular carcinoma $(n=33)$ & $42(24)[7-120]$ \\
Cholangiocellular carcinoma $(n=4)$ & $59(42)[40-100]$ \\
Lymphoma $(n=4)$ & $44(17)[17-60]$ \\
Haemangioendotheliosarcoma $(n=1)$ & 100 \\
\hline Values are mean (SD) [range]. & \\
\hline
\end{tabular}

With PIUS imaging, two separate $180^{\circ}$ out of phase pulses are transmitted into the tissue. The linear echoes reflected from the tissue are cancelled when the received signals are summed whereas the non-linear echoes reflected from the microbubbles are not, forming the final detectable US signal.

\section{PIUS technique}

After completion of the baseline scan, Levovist was injected intravenously $(400 \mathrm{mg} / \mathrm{ml})$ into the cubital vein using a 20 gauge needle at a bolus dose of $1 \mathrm{ml} / \mathrm{s}$ followed by a $10 \mathrm{ml}$ normal saline flush. The dose per injection was $4 \mathrm{~g}$ in all patients. Most patients received one injection but two injections were required in 15 patients with benign lesions and in 13 patients with liver cirrhosis and suspected HCC due to technical problems. The delayed contrast enhanced PIUS scan was started two minutes after contrast injection (to avoid bubble destruction) with a high mechanical index, focusing on the lesions to generate stimulated acoustic emissions. For the PIUS scans, ensemble contrast imaging software was used with the following settings: insonating frequency $2.5 \mathrm{MHz}$; parallel processing; single focal zone with depth adapted to the liver lesion; mechanical index 1.21.7; and frame rate $>10(-15) /$ second. When an individual sweep was completed, the image was frozen and the individual frames of the sweep were reviewed on the cine loop without time constraints. The delayed intermittent contrast enhanced phase inversion scanning was terminated within 10 minutes.

All examinations were digitally stored/documented by a commercially available system (E\&L, Erlangen, Germany) and some examinations were additionally stored on magnetic optical discs and S-VHS video tapes. The images of a particular patient were always shown to the second reader in the same sequence, baseline followed by PIUS (image).

\section{Histological evaluation}

Biopsy under US guidance was performed with a Temno $1.2 \mathrm{~mm}$ cutting needle to allow accurate histological evaluation. Liver biopsy specimens were fixed in formalin and embedded in paraffin for routine staining and immunohistochemistry. In two patients more than one biopsy was necessary to determine the correct histological nature of the lesion.

\section{Statistics}

Clinical characteristics of the patients are expressed as mean (SD) and median values (with limits of $50 \%$ interquartile ranges) when appropriate. Sensitivity and specificity were calculated for identification of malignant lesions using the $\chi^{2}$ test and $\mathrm{p}<0.05$ was considered to be statistically significant. Sensitivity was defined as the percentage of malignant lesions properly classified as malignant by US. Specificity was defined as the percentage of benign lesions properly classified as benign by US.

\section{RESULTS \\ Adequate examinations}

In all patients the contrast enhancement penetrated deep enough to examine the investigated the liver lesion. In 13 patients with liver cirrhosis and in 15 patients with benign lesions, disagreement between the two readers occurred with respect to the image quality after the first bolus injection due to inhomogeneous enhancement. Repeated contrast injection and optimisation of the examination technique and software settings (focus, depth penetration, intercostal/subcostal approach) lead to adequate examination results in all 28 patients. The enhancement was limited to the focal zone or depth penetration of $10-15 \mathrm{~cm}$ (using a variable focal zone) and did not always reach all parts of the liver. 


\section{Contrast enhanced US in the Levovist portal venous and late phase}

In all 79 patients with malignant liver lesions, hypoechoic contrast enhancement of the lesion was detected in the portal venous and late phase after Levovist injection, indicating a sensitivity of $100 \%$. Other lesions such as cysts and calcifications were also documented but were not included in the analysis. In contrast with the invariably pronounced hypoechoic metastases during the portal venous and liver specific late phase, the signal voids of HCC were variable and less impressive in 13 patients with heterogenous enhancing liver parenchyma during the portal venous and liver specific late phase, also indicating that HCC may show contrast uptake which may lead to misinterpretation (table 2). In 79 patients a malignant liver lesion was histologically diagnosed: metastatic liver disease in 37 patients (fig 1), HCC in 33 patients (all HCC were found in a cirrhotic liver; 21 patients were classified as Child-Pugh stage A and 12 patients as stage B; the aetiology was virus related in 15 patients, haemochromatosis related in one patient, and alcohol related in 17 patients), cholangiocellular carcinoma in four patients, lymphoma in four patients (Hodgkin's lymphoma $(n=1)$, non-Hodgkin's lymphoma $(n=3))$, and haemangioendotheliosarcoma in one patient. In three patients more than one lesion had been biopsied, revealing haemangioma and non-Hodgkin's lymphoma in one, adenoma and HCC in two (one of those has already been published $\left.{ }^{10}\right)$, and focal nodular hyperplasia (FNH), haemangioma, and haemangioendotheliosarcoma in another patient. Patients with metastatic liver disease had the following histology: adenocarcinoma $(\mathrm{n}=24$; primary site: colorectal $(n=20)$ and pancreatic $(n=4))$, small cell carcinoma of the lung $(n=3)$, non-small cell carcinoma of the lung $(n=1)$, neuroendocrine carcinoma $(n=6)$, melanoma $(n=2)$, and breast cancer $(n=1)$. In some patients with histologically proven malignant liver disease, we found previously undetected lesions but analysing these lesions was not subject of the study.

In 95 patients, a benign liver lesion was diagnosed by liver biopsy and histology: FNH in 36 patients, haemangioma in 31 patients, adenoma in 10 patients, microhamartomas (von Meyenburg complexes) in four patients, abscesses in five patients, regional fatty infiltration in four patients, hyperregenerative nodule, nodular regenerative hyperplasia, inflammatory pseudotumour of the liver, bacillary angiomatosis, ${ }^{11}$ and focal biliary cirrhosis in one patient. Other lesions such as cysts and calcifications were also documented but were not included in the analysis. In 35/36 patients with FNH, in all 31 patients with haemangioma, in all 10 patients with adenomas, in all four patients with microhamartomas (von Meyenburg complexes), in all four patients with regional

Table 2 Contrast enhancement in 74 patients with histologically proven malignant liver tumours or lesions

\begin{tabular}{ll}
\hline Liver tumour & $\begin{array}{l}\text { Hypoechoic contrast } \\
\text { enhancement }\end{array}$ \\
\hline Malignant liver lesions $(n=79)$ & $79 / 79$ \\
Metastatic liver tumour $(n=37)$ & $37 / 37$ \\
Hepatocellular carcinoma $(n=33)^{*}$ & $33 / 33$ \\
Cholangiocellular carcinoma $(n=4)$ & $4 / 4$ \\
Lymphoma $(n=4)$ & $4 / 4$ \\
Haemangioendotheliosarcoma $(n=1)$ & $1 / 1$ \\
\hline
\end{tabular}

*Hepatocellular carcinomas were variable and less impressive in 13 patients with a heterogeneous enhancing liver parenchyma during the liver specific late phase, also indicating that hepatocellular carcinomas may show some contrast uptake which might lead to misinterpretation. In those 13 patients, repeated contrast examinations were necessary to determine the malignant nature of the lesion due to technical reasons.
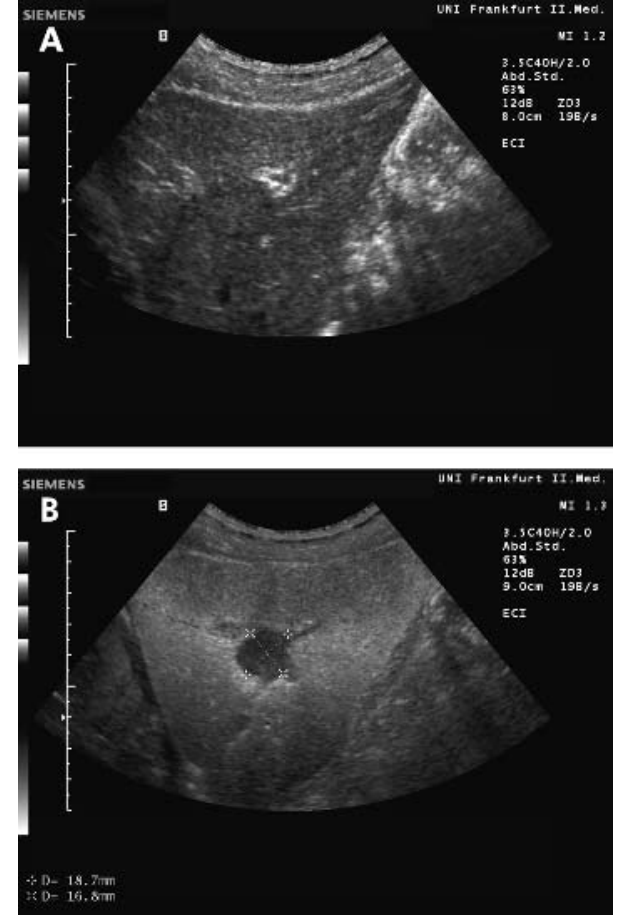

Figure 1 Malignant liver lesion in a patient with colorectal carcinoma and histologically proven liver metastasis. The unenhanced baseline scan shows a slightly heterogeneous liver parenchyma and one focal lesion in the left lobe of the liver (A). Phase inversion ultrasound in the late phase after contrast administration revealed a typical hypoechoic lesion, indicating malignancy (B).

fatty infiltration, and in patients with hyperregenerative nodule, nodular regenerative hyperplasia, inflammatory pseudotumour of the liver, bacillary angiomatosis, and focal biliary cirrhosis, but in none of the five patients with abscesses, homogenous contrast enhancement of the lesion in the portal venous and late phase after Levovist injection was observed (table 3 ).

In summary, hypoechoic contrast enhancement surrounded by enhanced liver parenchyma as a predictive sign of malignancy had $100 \%$ sensitivity in detecting malignancy. Homogeneous Levovist enhancement in the portal venous and late phase had $93 \%$ specificity as an indicator of benign disease.

\section{Characterisation of individual liver tumours}

The homogenous contrast enhancement pattern of FNH characteristically exceeded that of normal liver parenchyma (fig 2), in contrast with the isoechoic (or slightly hypoechoic) appearance in the portal venous and liver specific late phase of liver cell adenoma, with variable behaviour depending on the size and regressive features.

The vascular phase contrast enhancement pattern of haemangiomas was variable. One characteristic was peripheral nodular enhancement with progressive heterogeneous centripetal fill in, with considerable enhancement in the portal venous and liver specific late phase imaging, revealing diminished contrast to the surrounding liver parenchyma in all patients. Areas with thromboses and calcifications were noticed, associated with an increased size of lesions.

\section{DISCUSSION}

Accurate detection and characterisation of liver lesions with acoustic properties similar to those of the surrounding normal liver parenchyma has always been a significant 
Table 3 Contrast enhancement in 95 patients with histologically proven benign liver tumours or lesions

\begin{tabular}{ll}
\hline Liver tumour & $\begin{array}{l}\text { Isoechoic contrast } \\
\text { enhancement }\end{array}$ \\
\hline Benign liver lesions $(n=95)$ & $88 / 95$ \\
Focal nodular hyperplasia $(n=36)$ & $35 / 36$ \\
Haemangioma $(n=31)$ & $31 / 31^{*}$ \\
Adenoma $(n=10)$ & $10 / 10$ \\
Abscess $(n=5)$ & $0 / 5$ \\
Microhamartomas $(n=4)$ & $4 / 4$ \\
Focal steatosis $(n=4)$ & $4 / 4$ \\
Focal biliary cirrhosis $(n=1)$ & $1 / 1$ \\
Bacillary angiomatosis $(n=1)$ & $1 / 1$ \\
Nodular regenerative hyperplasia $(n=1)$ & $1 / 1$ \\
Regenerative nodule $(n=1)$ & $1 / 1$ \\
Inflammatory pseudotumour $(n=1)$ & $0 / 1$ \\
\hline
\end{tabular}

*The vascular phase contrast enhancement pattern of haemangiomas was variable. Characteristic was a progressive heterogeneous centripetal fill in and considerable enhancement on liver specific late phase imaging, revealing diminished contrast to the surrounding liver parenchyma in all patients.

limitation of conventional grey scale imaging. Brightness (B) mode ultrasonography is highly sensitive and specific in characterising cysts and calcifications, leading to a definitive diagnosis, but showed several limitations in patients with primary and secondary liver tumours. It was recently shown that contrast enhanced US using contrast specific non-linear imaging techniques improved the detection rate of liver metastases in comparison with B mode US, and had a detection rate similar to that reported for CT and MRI techniques. ${ }^{3-6}$ In these recently published detection studies in patients with known malignancies using Levovist in the portal venous and liver specific late phase, additional lesions were found in $40-45 \%$ of patients. Furthermore, it was recently shown that lower interobserver variability was found in contrast enhanced sonography than in baseline sonography. ${ }^{6}$

Characterisation of liver lesions starts once an abnormality is found. When an imaging procedure can detect liver masses, it should at least be able to differentiate between benign and malignant lesions, as benign and malignant lesions were reported to vary in their uptake during the portal venous and liver specific late phase after injection of Levovist. ${ }^{12}$ Although the precise mechanism of the portal venous and liver specific late phase accumulation of Levovist in a normal liver is unknown, the temporal course and distribution was suggested because of interaction with the sinusoids and reticuloendothelial system. ${ }^{3}$ We speculate that the non-enhancing defects of malignant liver lesions, but also abscesses, might be explained by the lack of liver specific tissue-for example, portal veins, sinusoids, and reticuloendothelial cells. The degree of late phase uptake by a lesion is mainly determined by the degree of similarity of the lesion to normal liver parenchyma, resulting in false positive findings and misinterpretation in patients with abscesses, necrosis, scars, calcifications, cysts, and thromboses. As cysts and calcifications may be correctly analysed by conventional B mode US, this is therefore mandatory before using US contrast agents. The importance of this dual imaging approach (conventional B mode US/contrast enhanced ultrasonography) is highlighted by the recognition of the high prevalence of benign liver lesions in the adult population, which in the vast majority do not require treatment, such as cysts, calcifications, and most benign liver tumours. Therefore, correct characterisation of the primary imaging method, US, is crucial for further diagnostic, therapeutic, and prognostic implications.
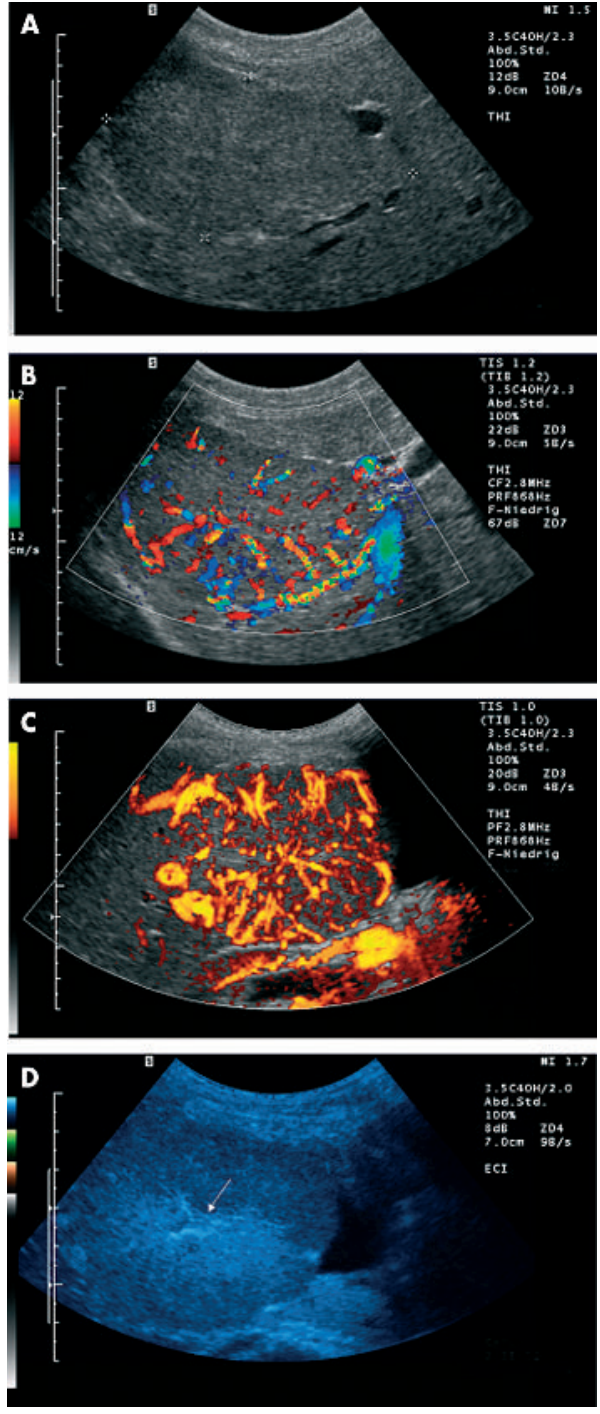

Figure 2 Benign liver lesion in a young woman with histologically proven focal nodular hyperplasia. The unenhanced conventional $B$ mode (A), colour Doppler (B), and power Doppler (C) imaging study shows a hypervascular liver tumour. Phase inversion ultrasound after contrast administration shows isoechoic liver parenchyma in the late phase (D). Arrow: central artery and scar.

The results of the present study show that contrast enhanced phase inversion sonography may discriminate in the portal venous and liver specific late phase between benign liver specific tissue and non-liver specific tissue, mainly malignant focal liver lesions. There were only a few false positive findings, mainly caused by abscesses and necrosis, and two liver tumours-one case of old FNH with mainly scar tissue, and one case of inflammatory pseudotumour of the liver, which was definitively diagnosed only by operation. The difficult diagnosis of the inflammatory pseudotumour of the liver is in accordance with current literature.

In contrast, homogeneous uptake was found in FNH, adenoma, haemangioma, focal steatosis, hyperregenerative nodules, and other benign liver tumours and nodules. Therefore, homogeneous parenchymal Levovist uptake in the portal venous and liver specific late phase seems to be indicative of benign focal liver lesions. In the present study, none of the lesions with homogeneous Levovist uptake in the portal venous and liver specific late phase was found to be malignant. This is in accordance with recently published 
studies which mainly used imaging methods as the gold standard but not histology in all patients. It is important clinically that there were no false negative findings with respect to malignant liver lesions.

Microbubble derived US contrast agents were originally developed to enhance Doppler signals during the early vascular phase. The dynamic profile of these contrast enhancing agents is similar to contrast media used by CT and MRI imaging. Additionally, Levovist accumulates in normal liver parenchyma during a liver specific late phase. This late phase contrast effect is specific to normal liver parenchyma and spares focal lesions with non-liver tissue. ${ }^{13-15}$

The late phase sinusoidal contrast effect cannot be detected using conventional (fundamental) B mode US. This requires highly sensitive microbubble specific imaging techniques that utilise the non-linear (that is, distorted) signals that are returned from microbubbles as a result of harmonic resonance and-most relevant-in the case of focal zone dependent Levovist microbubble destruction with stimulated acoustic emission.

Despite the obvious advantage of improving the sonographic diagnosis of liver lesions, contrast enhanced portal venous and late phase US has some problems. Firstly, only high end US machines are equipped for this procedure. Secondly, several pitfalls need to be considered by the investigator in obtaining correct results. Important pitfalls are, for example, destruction of Levovist by continuous insonation, putative destruction of Levovist during bolus injection by using a needle smaller than 21 gauge or the valve of the needle, ${ }^{16}$ or incorrect setting of the focal zone or mechanical index.

Liver tumours and nodules may vary greatly in their behaviour during the different vascular phases, and also depending on the degree of neovascularisation. In the present study, we focused on the portal venous and liver specific late phase. Better characterisation of lesions than differentiation of benign from malignant may occur by also analysing the arterial phase, but this was not the subject of our study.

In conclusion, contrast enhanced PIUS in the portal venous and liver specific late phase after injection of Levovist considerably improves the characterisation of liver tumours compared with conventional B mode sonography, leading to differentiation of benign and malignant liver lesions in most patients, if cysts and calcifications are excluded by conventional B mode ultrasonography. PIUS facilitates the clinical decision as to whether or not a sonographically detected liver lesion will need further investigation. From this point of view, the new technique may help to reduce unnecessary or invasive examinations in certain cases, such as invasive liver biopsy, CT scan, and MRI.

\section{Authors' affiliations}

C F Dietrich, A Ignee, G Schuessler, Second Department of Internal Medicine, Caritas Hospital Bad Mergentheim, Germany

J Trojan, Second Department of Internal Medicine, Johann Wolfgang

Goethe University Medical Centre, Frankfurt am Main, Germany

C Fellbaum, Senckenberg Centre of Pathology, Johann Wolfgang

Goethe University Medical Centre, Frankfurt am Main, Germany

\section{REFERENCES}

1 Wernecke K, Rummeny E, Bongartz G, et al. Detection of hepatic masses in patients with carcinoma: comparative sensitivities of sonography, $\mathrm{CT}$, and MR imaging. AJR Am J Roentgenol 1991;157:731-9.

2 Clarke MP, Kane RA, Steele G ir, et al. Prospective comparison of preoperative imaging and intraoperative ultrasonography in the detection of liver tumors. Surgery 1989;106:849-55.

3 Albrecht T, Blomley MJK, Burns PN, et al. Improved detection of hepatic metastases with pulse-inversion US during the liver-specific phase of SHU 508A: Multicenter study. Radiology 2003;227:361-70.

4 Harvey $\mathrm{CJ}$, Blomley MJ, Eckersley RJ, et al. Pulse-inversion mode imaging of liver specific microbubbles: improved detection of subcentimetre metastases. Lancet 2000;355:807-8.

5 Albrecht T, Hoffmann CW, Schmitz SA, et al. Phase-inversion sonography during the liver-specific late phase of contrast enhancement: improved detection of liver metastases. AJR Am J Roentgenol 2001;176:1191-8.

6 von Herbay A, Vogt C, Haussinger D. Late-phase pulse-inversion sonography using the contrast agent levovist: differentiation between benign and malignant focal lesions of the liver. AJR Am J Roentgenol 2002;179:1273-9.

7 Dietrich CF, Lee JH, Herrmann G, et al. Enlargement of perihepatic lymph nodes in relation to liver histology and viremia in patients with chronic hepatitis C. Hepatology 1997;26:467-72, (erratum in Hepatology 1998;28:1444).

8 Dietrich CF, Lee JH, Gottschalk R, et al. Hepatic and portal vein flow pattern in correlation with intrahepatic fat deposition and liver histology in patients with chronic hepatitis C. AJR Am J Roentgenol 1998;171:437-43.

9 Dietrich CF, Chichakli M, Hirche TO, et al. Sonographic findings of the hepatobiliary-pancreatic system in adult patients with cystic fibrosis. $J$ Ultrasound Med 2002;21:409-16.

10 Gossmann J, Schevermann EH, Frilling A, et al. Multiple adenomas and hepatocellular carcinoma in a renal transplant patient with glycogen storage disease type la (von Gierke disease). Transplantation 2001 72:343-4.

11 Braden B, Helm B, Fabian T, et al. Bacillary angiomatosis of the liver, a suspected ultrasound diagnosis? Z Gastroenterol 2000;38:785-9.

12 Blomley MJ, Sidhu PS, Cosgrove DO, et al. Do different types of liver lesions differ in their uptake of the microbubble contrast agent $\mathrm{SH} U$ 508A in the late liver phase? Early experience. Radiology 2001;220:661-7.

13 Blomley MJ, Albrecht T, Cosgrove DO, et al. Improved imaging of liver metastases with stimulated acoustic emission in the late phase of enhancement with the US contrast agent SH U 508A: early experience. Radiology 1999;210:409-16.

14 Blomley M, Albrecht T, Cosgrove D, et al. Stimulated acoustic emission in liver parenchyma with Levovist. Lancet, 1998 21, 351:568.

15 Albrecht T, Hoffmann CW, Schettler S, et al. B-mode enhancement at phaseinversion US with air-based microbubble contrast agent: initial experience in humans. Radiology 2000;216:273-8.

16 Kratzer W, Kachele V, Merkle E, et al. Contrast enhanced power Doppler sonography: comparison of various administration forms of the ultrasound contrast agent Levovist. Rofo Fortschr Geb Rontgenstr Neven Bildgeb Verfahr 2000;172:443-8, (erratum in Rofo Fortschr Geb Rontgenstr Neuen Bildgeb Verfahr 2000;172:947) 